\title{
Problem of modelling a layered medium taking into account the creep of its materials
}

\author{
Tatiana Bobyleva ${ }^{1, *}$, and Alexei Shamaev ${ }^{2,3}$ \\ ${ }^{1}$ Moscow State University of Civil Engineering, Yaroslavskoye Shosse, 26, Moscow, 129337, Russia \\ ${ }^{2}$ Ishlinsky Institute for Problems in Mechanics of the Russian Academy of Sciences, Pr. \\ Vernadskogo, 101-1, Moscow, 119526, Russia \\ ${ }^{3}$ Lomonosov Moscow State University, GSP-1, Leninskie Gory, Moscow, 119991, Russia
}

\begin{abstract}
Problems for heterogeneous materials arise in many areas of construction. This article deals with elastic-creeping layered composite materials. Relations of the theory of linear heredity of Boltzmann-Volterra are accepted. Creep kernels are given by the sum of a finite number of decreasing exponential functions. Such sum of several exponential functions can be used to approximate the experimental data for the creep and relaxation kernels with a given accuracy. It is obtained that the resulting homogeneous medium is described by a creep kernel, which is also a sum of decreasing exponential functions. Algorithm for the rapid and accurate calculation of averaged creep kernels of a homogenized material is proposed. Some qualitative properties of the received homogenized model are analyzed.
\end{abstract}

\section{Introduction}

The study of the stress-strain state of a system of plane-parallel layers of various materials has important applications in the mechanics of composites, structural mechanics, rock mechanics, and soils. A mathematical description of such media usually contains integrodifferential equations with rapidly oscillating coefficients, so direct investigation of such problems is rather complicated.

The article suggests a method for modelling the stress-strain state of layered materials. This method is based on a combination of the theory of averaging [1-4] and creep theory $[5,6]$. As an example, the problem of averaging an inhomogeneous elastic medium with the use of theoretical results was solved in [7]. Averaged models describing the joint motion of the layers consisting of elastic and viscoelastic materials [8], and also of two creeping materials [9], were constructed. In paper [10] effective elastic modules of a layered elasticcreeping medium were obtained. Estimates of the closeness of the displacement and stress fields for the initial and averaged models are given in [11], and an example of numerical modelling of the characteristics of an averaged material is presented. Averaging problems in hereditary mechanics for materials with periodic structure were considered in [12].

\footnotetext{
* Corresponding author: tatyana2211@outlook.com
} 
In the article as the main rheological relations, the relations of the theory of linear heredity of Boltzmann-Volterra are accepted, according to which the deformation $e(t)$ at time $t$ has the form

$$
e(t)=\frac{1}{E}\left[\sigma(t)+\int_{0}^{t} K(t-\tau) \sigma(\tau) d \tau\right],
$$

where $E$ is the Young's modulus, $\sigma$ is the stress, $K(t-\tau)$ denotes the creep kernel, which is a decreasing function [5].

Parameters of the rheological model are determined on the basis of laboratory tests. Finite sums of the exponential functions of time are taken as the creep kernels of each layer. Experiments prove that such functions are a good way to describe the creep of materials over a long period of time. Such kernels are called spectral.

It is clear that models may be very different for the various materials used in engineering practice, such as metals and alloys, polymers, fiber composites (with polymer or metal matrix), concrete and wood. However, to a large extent it is possible to employ the same principles and concepts in establishing constitutive relations for these different materials.

It is well known that in the modelling of elastic-creeping or viscoelastic materials with the help of combinations of springs and dashpots, the transfer function (the multiplier that relates the Laplace transforms of stresses and deformations) is the sum of the simplest fractions with coefficients of the same sign [6]. The purpose of this paper is to investigate the problem of saving this transfer function's property for the averaged model of a layered composite.

\section{Problem specification and decision}

We consider a region $\Omega$ which consists of layers of elastic-creeping isotropic materials. These layers alternate in pairs. Let $\varepsilon$ be the length ratio of two adjacent layers to the characteristic size of the sample, and the thicknesses of each individual layer are respectively equal to: $\varepsilon_{1}=\varepsilon h$ and $\varepsilon_{2}=\varepsilon(1-h), 0 \leq h \leq 1$. Cartesian coordinate system $O x_{1} x_{2} x_{3}$ is chosen. All the layers of the considered region $\Omega$ are parallel to the $O x_{1} x_{2}$ coordinate plane as shown in Figure 1.

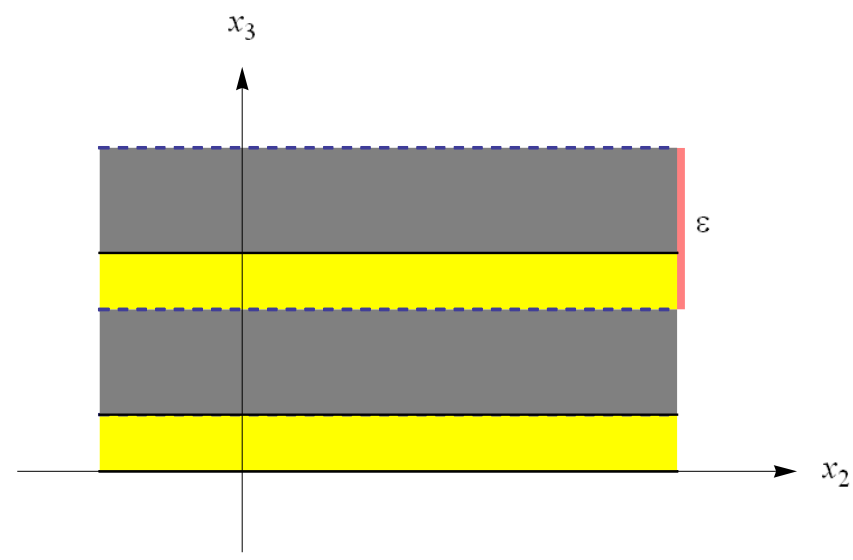

Fig. 1. Layered structure of region, $\varepsilon$ is the characteristic cell size of periodicity 
We consider the elastic-creeping material therefore components of the stress tensor $\sigma_{i j}(i, j=1 \div 3)$ are determined both at a given time and by the entire history of the body deformation. Therefore, the equations of state connecting the components of the strain and stress tensors for each layer are as follows $[5,6]$ :

$$
\sigma_{i j}^{(s)}=b_{i j k h}^{(s)} * e_{k h}^{(s)},
$$

here $\quad b_{i j k h}=c_{i j k h} \delta(t)+d_{i j k h}, e_{k h}^{(s)}=\frac{1}{2}\left(\frac{\partial u_{k}^{(s)}}{\partial x_{h}}+\frac{\partial u_{h}^{(s)}}{\partial x_{k}}\right),(k, h=1 \div 3)(s=1,2 \quad$ is the layer number), $u_{k}$ are components of the displacement vector, $c_{i j k h}^{(s)}$ are components of the elastic modulus tensor, $\delta(t)$ is Dirac-delta, $d_{i j k h}^{(s)}(t, \tau)$ are Volterra integral operators, namely,

$$
d_{i j k h} * e_{k h}=\int_{0}^{t} d_{i j k h}(t-\tau) e_{k h}(\tau) d \tau,
$$

variable $t$ specifies time. (Einstein convention for repeated indices is used.) Relaxation kernels $d_{i j k h}(t-\tau)$ depend on the difference $(t-\tau)$ because, by assumption, stress components $\sigma_{i j}$ are invariants with respect to the origin of time $t$. We take the sums of exponential functions as the relaxation kernels.

The ideal contact conditions are assumed on the horizontal surfaces of the layers, namely: components of the displacement and the components of stress parallel the $x_{3}$-axis are continuous, i.e. $\left[u_{i}\right]=0,\left[\sigma_{i 3}\right]=0,(i=1 \div 3)$.

Equilibrium equations in the theory of elasticity have the form [3]:

$$
\frac{\partial \sigma_{i j}(\mathbf{x}, t)}{\partial x_{j}}=f_{i}(\mathbf{x}, t) \text {. }
$$

In (4) we designated: $\mathbf{x}=\left(x_{1}, x_{2}, x_{3}\right)$, and $f_{i}(\mathbf{x}, t)$ are components of a vector of external forces.

Components of the elastic tensor and relaxation kernel tensors in (2) for isotropic materials have the form [6]:

$$
\begin{gathered}
c_{i j k h}=\lambda \delta_{i j} \delta_{k h}+\mu\left(\delta_{i k} \delta_{j h}+\delta_{i h} \delta_{j k}\right), \\
d_{i j k h}=-\left(D_{v}(t)-\frac{1}{3} D_{s h}(t)\right) \delta_{i j} \delta_{k h}-\frac{1}{2} D_{s h}(t)\left(\delta_{i k} \delta_{j h}+\delta_{i h} \delta_{j k}\right) .
\end{gathered}
$$

We denote here by $D_{s h}$ and $D_{v}$ the regular part of the shear and the bulk relaxation respectively, by $\delta_{i j}$ Kronecker symbol. Suppose that the amplitude of a bulk relaxation kernel is proportional to the amplitude of the shear relaxation kernel with a proportionality coefficient $k_{s}$ for each layer: $\left(D_{v}\right)_{s}=k_{s}\left(D_{s h}\right)_{s}, k_{s}$ is a constant, $k_{s}>0(s=1,2)$. Further, $D_{\text {sh }}$ is denoted by $D$. 
Since this array is a layered medium all elastic modulus and relaxation kernels are periodic functions of the coordinate $z=\frac{x_{3}}{\varepsilon}$ ( $\varepsilon$ is the relative cell period) and are piecewise constant functions of this variable, i.e., elastic modulus and relaxation kernels have the form [10]:

$$
\lambda(z)=\left\{\begin{array}{l}
\lambda_{1}, z \in[0 ; h] \\
\lambda_{2}, z \in[1-h ; 1]
\end{array} \quad \mu(z)=\left\{\begin{array}{l}
\mu_{1}, z \in[0 ; h] \\
\mu_{2}, z \in[1-h ; 1]
\end{array} \quad D(z, t)=\left\{\begin{array}{l}
D_{1}(t), z \in[0 ; h] \\
D_{2}(t), z \in[1-h ; 1]
\end{array} .\right.\right.\right.
$$

In (7) $\lambda_{s}, \mu_{s}$ are Lame parameters for each layer. We choose creep kernel as the sum of exponential functions for each layer ( $s$ is the layer number, $s=1,2$ ):

$$
D_{s}(t)=\sum_{k=1}^{M} c_{k s} \exp \left(-\alpha_{k s} t\right)
$$

$c_{k s}, \alpha_{k s}$ are constants, $c_{k s}<0, \alpha_{k s}>0$ [6]. As experiments show, such kernels describe well the behavior of relaxation materials for long periods of time.

Consider an algorithm for constructing the averaged characteristics of layered elasticcreeping medium. We have the integral equation

$$
q(t)+\int_{0}^{t} K(t-\tau) q(\tau) d \tau=f(t)
$$

in which

$$
K(t)=\sum_{k=1}^{M} c_{k} e^{-\alpha_{k} t}, f(t)=\sum_{k=1}^{M} f_{k} e^{-\beta_{k} t} .
$$

We apply the Laplace transform in the time domain to the equation (9)

$$
\tilde{f}(p)=\int_{0}^{\infty} f(t) e^{-p t} d t
$$

As a result, we obtain

$$
\tilde{q}(p)+\tilde{K}(p) \tilde{q}(p)=\tilde{f}(p), \text { or } \tilde{q}(p)=\frac{\tilde{f}(p)}{1+\tilde{K}(p)} .
$$

Further, since $K(t)$ and $f(t)$ are represented as sums of exponential functions (10), we get

$$
\tilde{q}(p)=\sum_{k=1}^{M} \frac{f_{k}}{p+\beta_{k}}\left(1+\sum_{k=1}^{M} \frac{c_{k}}{p+\alpha_{k}}\right)^{-1}
$$




$$
\left(1+\sum_{k=1}^{M} \frac{c_{k}}{p+\alpha_{k}}\right)=\frac{\prod_{k=1}^{M}\left(p+\alpha_{k}\right)+\sum_{j=1}^{M} \prod_{\substack{k=1 \\ j \neq k}}^{M}\left(p+\alpha_{k}\right) c_{j}}{\prod_{k=1}^{M}\left(p+\alpha_{k}\right)}=\frac{p P_{M-1}(p)+\sum_{\substack{j=1 \\ k=1}}^{M} \prod_{\substack{k=1 \\ j \neq k}}^{M} \alpha_{k} c_{j}+\prod_{k=1}^{M} \alpha_{k}}{\prod_{k=1}^{M}\left(p+\alpha_{k}\right)},
$$

here $P_{M-1}(p)$ is a polynomial of degree $M-1$. Suppose that all coefficients $c_{k}$ $(k=1, \ldots, M)$ are negative: $c_{k}<0$. Such assumption is completely justified by real experimental data on the behavior of the relaxation kernels. Then the graph of the function $r(p)=\sum_{k=1}^{M} \frac{c_{k}}{p+\alpha_{k}}$ is as in the Figure 2.

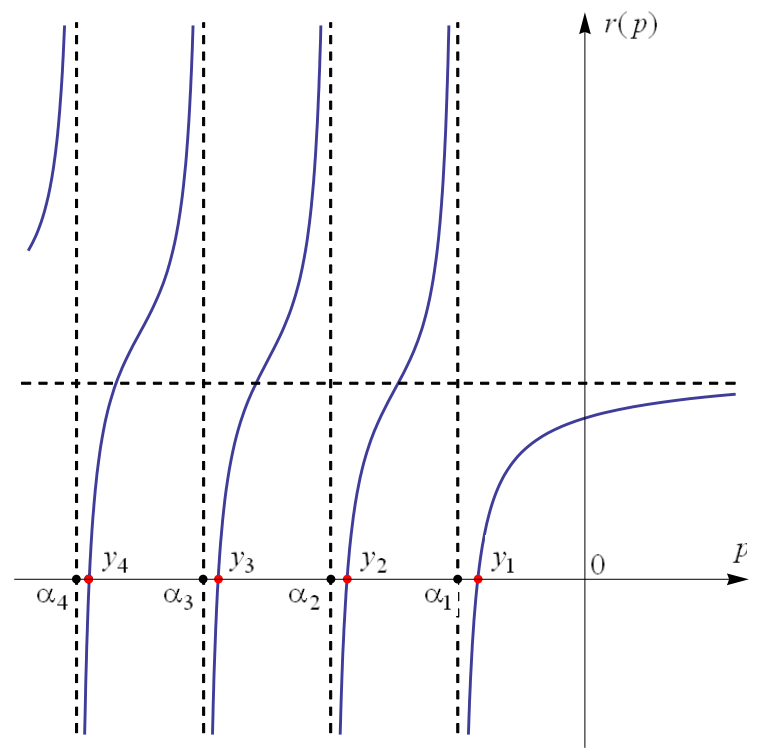

Fig. 2. Zeros $y_{k}$ and poles $\alpha_{k}(k=1 \div 4)$ of the function $r(p)$

It is obvious that the function $1+r(p)$ (this is the ratio of polynomials of degree $M$ ) has $M$ real roots $-v_{1},-v_{2}, \ldots,-v_{M}$. The roots $-v_{k}$ lie between the numbers $-\alpha_{k},-\alpha_{k-1}$ (this follows from sign-definiteness of $c_{k}$ ), and can easily be found by simple algorithms (for example, by method of half division). The root $-v_{1}$ does not lie between $-v_{k},-v_{k-1}$, however, it can be found by Vieta's theorem:

$$
-v_{1}=(-1)^{M+1}\left(\sum_{\substack{j=1 \\ j \neq 1 \\ j \neq k}}^{M} \prod_{k} c_{j}+\prod_{k=1}^{M} \alpha_{k}\right)\left(v_{2} \cdot \cdots \cdot v_{M}\right)^{-1} .
$$

Consequently, the function $(1+q(p))^{-1}$ can be represented in the form 


$$
(1+q(p))^{-1}=\frac{l(p)}{\left(p+v_{1}\right)\left(p+v_{2}\right) \cdots\left(p+v_{M}\right)},
$$

here $l(p)$ is a polynomial of degree $M, l(p)=p^{M}+A_{1} p^{M-1}+\cdots+A_{M}$. Consider the function

$$
\begin{gathered}
\tilde{q}(p)=\sum_{k=1}^{M} \frac{f_{k}}{p+\beta_{k}}\left(1+\sum_{k=1}^{M} \frac{c_{k}}{p+\alpha_{k}}\right)^{-1}=\left(\sum_{k=1}^{M} \frac{f_{k}}{p+\beta_{k}}\right) \frac{l(p)}{\left(p+\alpha_{1}\right)\left(p+\alpha_{2}\right) \cdots\left(p+\alpha_{M}\right)}= \\
=\frac{m(p)}{\left(p+\beta_{1}\right) \cdots\left(p+\beta_{M}\right)\left(p+\alpha_{1}\right) \cdots\left(p+\alpha_{M}\right)},
\end{gathered}
$$

where $m(p)$ is a polynomial of degree $2 M$ with respect to a variable $p$. We represent $\tilde{q}(p)$ as the sum of the simplest fractions

$$
\frac{m(p)}{\left(p+\beta_{1}\right) \cdots\left(p+\beta_{M}\right)\left(p+\alpha_{1}\right) \cdots\left(p+\alpha_{M}\right)}=\sum_{k=1}^{M} \frac{A_{k}}{p+\beta_{k}}+\sum_{k=1}^{M} \frac{B_{k}}{p+\alpha_{k}},
$$

in which numbers $A_{k}$ and $B_{k}$ are to be determined. By reducing the right-hand side to the common denominator and equating terms with equal powers in the numerator, we obtain a linear system of order $2 M$. We define $A_{k}, B_{k}$ from this system. As a result, the solution of the integral equation (9) has the next form

$$
q(t)=\sum_{k=1}^{M} A_{k} e^{-\beta_{k} t}+\sum_{k=1}^{M} B_{k} e^{-\alpha_{k} t}
$$

Thus, the solution of the integral equation (9) (provided that in (10) all $c_{k}$ are negative) is reduced to:

- the definition by the simplest methods (for example, by means of the algorithm of division of a segment in half) of the roots $v_{2}, \ldots, v_{M}$;

- the determination by the formula (13) of the root $v_{1}$;

- the definition by solving a linear system of dimension $2 M$ numbers $A_{k}, B_{k}$ $(k=1, \ldots, M)$.

Finally, the solution of the integral equation (9) can be represented by the formula (19).

It should be noted that the order of "decay" of the solution of equation (9) can only be "less" than the right side of the it equation, and also it can only be "smaller" than the kernel of equation (9), since $-\alpha_{1} \leq-v_{1}$. The value $-v_{1}$ is determined by the formula (15). Thus, the additional condition $c_{k}<0(k=1, \ldots, M)$ enables fast and economical both in the number of operations and in memory of the numerical solution of equation (9).

Returning to the problem of constructing effective characteristics of a layered medium, we can state that in this way (for the original creep kernels), it is possible to solve integral equations quickly and accurately. And with their help, the effective characteristics of a layered medium are determined. We apply the Laplace transform (11) in the time domain to the equilibrium equations (4) with the assumption (2) and (3). The result is the system of elasticity theory with a complex parameter $p$. We apply homogenization method described 
in $[1,2]$ to this system. After this we obtain a homogeneous anisotropic medium with averaged (effective) modules [10]. The symmetric matrix of its effective coefficients is:

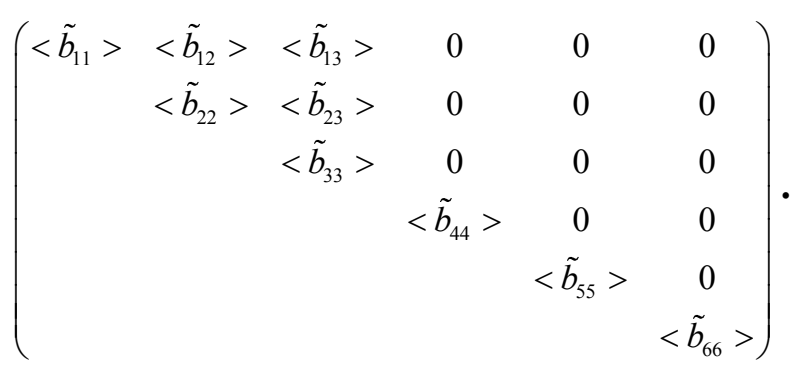

Here $\langle f\rangle=\int_{0}^{1} f(\xi) d \xi$ is denoted the averaging over the variable $\xi$.

Let $P=\sum_{k=1}^{M} \frac{c_{k}}{p+\alpha_{k}}, k_{s 1}=k_{s}+\frac{2}{3}, k_{s 2}=k_{s}-\frac{1}{3}$, then for isotropic materials $(5,6)$ elements of the matrix (20) have the following form:

$$
\begin{gathered}
<\tilde{b}_{11}>=<\tilde{b}_{22}>=<\lambda+2 \mu-k_{s 1} P>+\left\langle\frac{\lambda-k_{s 2} P}{\lambda+2 \mu-k_{s 1} P}\right\rangle^{2}<\left(\lambda+2 \mu-k_{s 1} P\right)^{-1}>^{-1}-\left\langle\frac{\left(\lambda-k_{s 2} P\right)^{2}}{\lambda+2 \mu-k_{s 1} P}\right\rangle \\
<\tilde{b}_{12}>=<\lambda-k_{s 2} P>+\left\langle\frac{\lambda-k_{s 2} P}{\lambda+2 \mu-k_{s 1} P}\right\rangle^{2}<\left(\lambda+2 \mu-k_{s 1} P\right)^{-1}>^{-1}-\left\langle\frac{\left(\lambda-k_{s 2} P\right)^{2}}{\lambda+2 \mu-k_{s 1} P}\right\rangle,(21) \\
<\tilde{b}_{13}>=\left\langle\frac{\lambda-k_{s 2} P}{\lambda+2 \mu-k_{s 1} P}\right\rangle<\left(\lambda+2 \mu-k_{s 1} P\right)^{-1}>^{-1}, \quad<\tilde{b}_{33}>=<\left(\lambda+2 \mu-k_{s 1} P\right)^{-1}>^{-1}, \\
\quad \tilde{b}_{44}>=<\tilde{b}_{55}>=<(\mu-0.5 P)^{-1}>^{-1}, \quad<\tilde{b}_{66}>=<\mu-0.5 P>.
\end{gathered}
$$

We know the boundaries of intervals containing the roots of polynomials, which are the desired indicators of the exponential kernels of the averaged operator. These indicators determine the creep parameters of the homogenized medium. They can be found by the wellknown fast and high-precision algorithm for dividing a segment in half. Let us estimate the number of these indicators. We use for this purpose the formulas (21). The averaging operation doubles the number of linear factors in the denominator of the corresponding fraction, and the multiplication operation also leads to a doubling of the number of linear factors in the denominator. A careful calculation shows that, in accordance with formulas (19), the number of indicators of exponents in homogenized kernels is estimated by $8 M$. And this number includes all the previous $2 M$ exponents of the materials of the individual layers. To calculate the coefficients for exponential functions of homogenized kernels, it is necessary to solve certain systems of linear algebraic equations. These systems arise when we decompose the ratio of two polynomials into the sum of the simplest fractions if the denominator roots are known. In our case, we can find these roots with the help of the abovementioned algorithm for dividing a segment in half. It should also be noted that in the presence of multiple root in the denominator, the inverse Laplace transform will give an exponential function with a polynomial factor, and not with a constant factor, as in the case of single roots. 


\section{Conclusion}

The method is proposed for finding the averaged characteristics of a layered elastic-creeping medium for the case of spectral (representing finite sums of decreasing exponential functions) kernels. For effective modules new exponent indicators can be easily found by half-division method, and the coefficients of exponential functions are found by solving systems of linear equations. The obtained technique can be used to calculate slow creeping displacements and stress-strain state of layered rocks, as well as to study a number of composite materials.

\section{References}

1. O.A. Oleynik, G.A. Iosif'yan, A.S. Shamaev, Mathematical problems in elasticity and homogenization (Elsevier, North-Holland, 1992)

2. D.I. Bardzokas, A.I. Zobnin, Mathematical modeling of physical processes in composite materials of periodic structure (URSS, Moscow, 2005)

3. B.E. Pobedrya, Mechanics of composite materials (MSU, Moscow, 1984)

4. R.M. Christensen, Mechanics of composite materials (Dover, New York, 2005)

5. Yu.N. Rabotnov, Elements of hereditary solid mechanics (Mir, Moscow, 1980)

6. A.A. Iyushin, B.E. Pobedrya, Foundations of the mathematical theory of thermoviscoelasticity (Nauka, Moscow, 1970)

7. T.N. Bobyleva, Procedia Engineering, 153, 103-106 (2016)

8. A.S. Shamaev, V.V. Shumilova, Proceedings of MIAS, 295 (1), 202-212 (2016)

9. A.S. Shamaev, V.V. Shumilova, Proceedings of MIAS, 295 (1), 213-224 (2016)

10. T.N. Bobyleva, A.S. Shamaev, Soil Mechanics and Foundation Engineering, 54 (4), 224-230 (2017)

11. T.N. Bobyleva, A.S. Shamaev, IFAC-PapersOnLine, 9th Vienna Int. Conf. on Math. Modelling, 51 (2), 138-143 (2018)

12. Yu. Orlik, Hindawi Publ. Corp. Abstract and Applied Analysis, 1-19 (2009) 